\title{
Diabetic Glomerulosclerosis*
}

J. M. B. BLOODWORTH, JR.

Department of Pathology, University of Wisconsin Medical School, Madison, and Madison Veterans Administration Hospital

I would like to show first the glomerulus of a 92-year-old man who did not have diabetes. It is perfectly normal. I want to emphasize that in the glomerulus there is not necessarily any ageing phenomenon per se. With the electron microscope there may be a slight increase in the thickness of the basement membrane of the glomerular capillary, but it is not visible by light microscopy.

In the normal glomerulus the afferent arteriole breaks up into capillary loops which spread out to form the lobules of the glomerulus. The central portion of each lobule or group of capillary loops is called the mesangial area (fig. 1). The capillary loops themselves are lined with endothelial cells, consisting of nuclei which protrude into the lumen, and a widely spread-out thin layer of fenestrated cytoplasm lining the capillary lumen. The endothelial cell is separated from the epithelial cell by a thin layer of basement membrane, and this basement membrane connects with little processes of similar material between adjoining mesangial cells. Outside the basement membrane are the epithelial cells, each with a wide extension of foot processes, forming a network around the capillary, with a tiny canaliculi between each foot process.

* Supported in part by grant \#AM07118 from the National Institutes of Health, U.S.P.H.S.; the Wisconsin Heart Association; U.S.P.H.S. institutional grant funds of the University of Wisconsin; and Veterans Administration research funds.
The mesangial area is the central area of each lobule and is separated from the blood by the endothelial cells. The mesangial cells are frequentìy surrounded by a little bit of basement membrane material. The mesangial cells have a more abundant cytoplasm and are better equipped with mitochondria and endoplasmic reticulum than the endothelial cells, but it seems likely that the mesangial cells are derived from the same group of cells as the endothelium. If endothelial cells are damaged, the mesangial cells can proliferate to form new endothelial cells. Also, I believe that the mesangial cells normally produce basement membrane material and pump it out in some fashion along the capillary wall to form the basement membrane of the periphery of the capillary loop. There are practically no mitochondria or endoplasmic reticulum in the cytoplasm of the flattened endothelial cells, and it seems very unlikely that they could be producing the basement membrane material. Others have suggested that the epithelial cells form basement membrane, but this seems unlikely as some capillaries do not have such cells.

In the diabetic, the mildest change is thickening of the basement membrane (fig. 2). The basement membrane of the normal capillary loop measures 2,000 to 3,000 $\AA$ in thickness, by our methods. In diabetics, the thickness is from 6,000 to $15,000 \AA$. There are occasional exceptions-diabetics who show only normal thickness of the glo- merular basement membrane-but the great majority of diabetics show a very definite thickening. In the early stages there is an abnormal accumulation of basement membrane material in the mesangial area. Gradually over many years this basement membrane material of the mesangial area increases until it appears to fill up the glomerulus. The material is PAS-positive. In the early stages, this lesion is diffuse glomerulosclerosis. This lesion is not specific for diabetes, and at this stage the pathologist cannot positively identify this as a diabetic process. It seems quite clear, however, that the development of nodular glomerulosclerosis, the Kimmelstiel-Wilson lesion which is specific for diabetes (fig. 3), is simply a further progression of the same process.

The little strands of basement membrane which normally separate the mesangial cells are called mesangial matrix. In the diabetic where this material is greatly increased, we call this material hyalinoid matrix. We now think it may be of a slightly different character. So we now have three basement membrane-like materials to think about in the glomerulus: basement membrane proper, the thin strands of normal mesangial matrix, and the pathologic material found in this area in diabetics, called hyalinoid matrix.

In the diabetic, as the basement membrane material accumulates, it piles up, particularly in the mesangial area, as hyalinoid matrix (fig. 2). This may form large 
masses or nodules in which mesangial cells appear to be trapped. This is the so-called KimmelstielWilson nodule of nodular glomerulosclerosis, and it is specific for diabetes. The basement membrane of Bowman's capsule also becomes thickened, and shows a laminated structure. It is of interest that the basement membrane of epithelial structures show a laminated thickening, whereas that of the capillaries usually shows a diffuse thickening.

In the beginning of this process, the foot processes of the epithelial cells are not affected. As diabetes progresses, the normal foot processes are progressively fused until they may be totally absent. The disappearance of foot processes is apparently associated with proteinuria, and this is found in the neophrotic syndrome from any cause. In the diabetic, the foot processes may not be lost until the Kimmelstiel-Wilson lesion is well advanced, and this correlates quite well with what we see clinically. The diabetic may go for years without proteinuria, then rather abruptly develop a nephrotic syndrome with heavy proteinuria, hypoalbuminemia, and edema. The Kimmelstiel-Wilson lesion itself probably has nothing to do with it. It is a reactive phenomenon of the glomerulus, with loss of the epithelial cell foot processes.

There are several other changes commonly seen in the kidney in diabetes. One is the so-called exudative lesion or hyaline-fibrinoid lesion. These occur in the glomerulus and are most often seen in far advanced diabetes. Similar lesions are seen in other diseases; in some of the collagen diseases for example. At times they appear as almost solid hyaline masses; at other times they have vacuoles or even become quite foamy. The first are composed of a hyaline material, while the latter contain a high percentage of fat. They may fill the capillaries adjacent to the Kimmelstiel-Wilson nodule. They occur beneath the thickened base- ment membrane of the capillaries, and in Bowman's capsule adjacent to the thickened basement membrane.

Another interesting phenomenon has been known for many years. Hypertension in the non-diabetic is associated with arteriolar hyalinization and sclerosis in the afferent arteriole of the glomerulus but not in the efferent. In diabetes -and as far as we know only in diabetes-when there is hypertension one finds hyalinization and arteriolar sclerosis not only in the afferent but also in the efferent arteriole. Whatever the pressure is within the glomerulus of the diabetic with hypertension, it must be higher than the pressure in the glomerulus of the hypertensive patient without diabetes.

There is also thickening of the basement membrane of the renal tubules in diabetes. It is important to realize that in any patient, atrophy of the tubules from any cause will be accompanied by thickening of the basement membrane. This is a nonspecific change. But in diabetes, the basement membrane of otherwise normal tubules becomes thickened. There is a lamination of this. As was mentioned above, the basement membrane of epithelial structures, when it becomes thickened in diabetes, shows a laminated structure.

Certain other findings in the diabetic kidney are well known. You

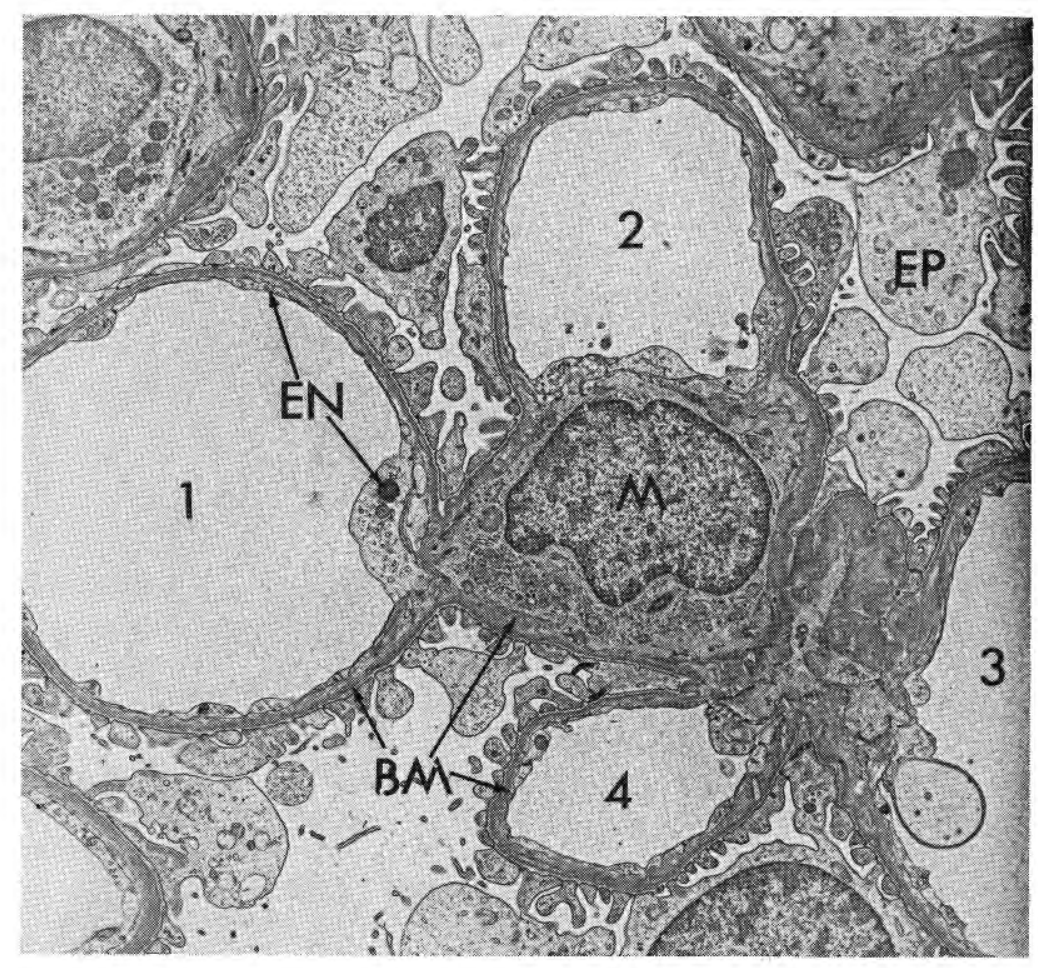

Fig. 1-Electron micrograph of a glomerular capillary tuft from a non-diabetic patient. The tuft is composed of a central mesangial area which is usually limited to a single mesangial cell $(M)$ surrounded by several capillary loops, in this case four. Each capillary lumen $(1,2,3,4)$ is surrounded by a very thin layer of endothelial cytoplasm $(E N)$ which is frequently fenestrated. The endothelial cytoplasm is, in turn, surrounded by a gray basement membrane $(B M)$. External to the basement membrane are various processes of the epithelial cells $(E P)$ which are called foot processes and are separated from each other by canaliculi $(4000 \times)$. 
are familiar with the yellow spots sometimes found in the medulla of the kidney in diabetes. This is renal papillary necrosis. It often occurs in association with pyelonephritis, but histologic examination shows that it is really a small infarct of the renal papilla, with necrosis in the center and inflammatory reaction around the edge. Although it may be associated with a highly virulent ascending pyelonephritis, there is obviously a vascular factor here. Pieces of infarcted and necrotic papilla can break off and be passed in the urine, and often these are sent to the pathologist for examination.

Pyelonephritis itself is a very common finding in diabetic pa- tients, as is well known. Mention should also be made of the glycogen deposition in the renal tubular cells, the Armanni-Ebstein lesion, formerly a pathologic hallmark of uncontrolled diabetes, but no longer the common finding it once was.

I would like to describe briefly my findings in experimentally-produced diabetes in dogs. Ten dogs were made diabetic, five by alloxan and five by injections of bovine growth hormone. The dogs were maintained on insulin, but in a severely diabetic state, for one to six years. All the animals manifested diffuse glomerulosclerosis, and seven developed nodules of the classical Kimmelstiel-Wilson type, as found in human diabetics. The

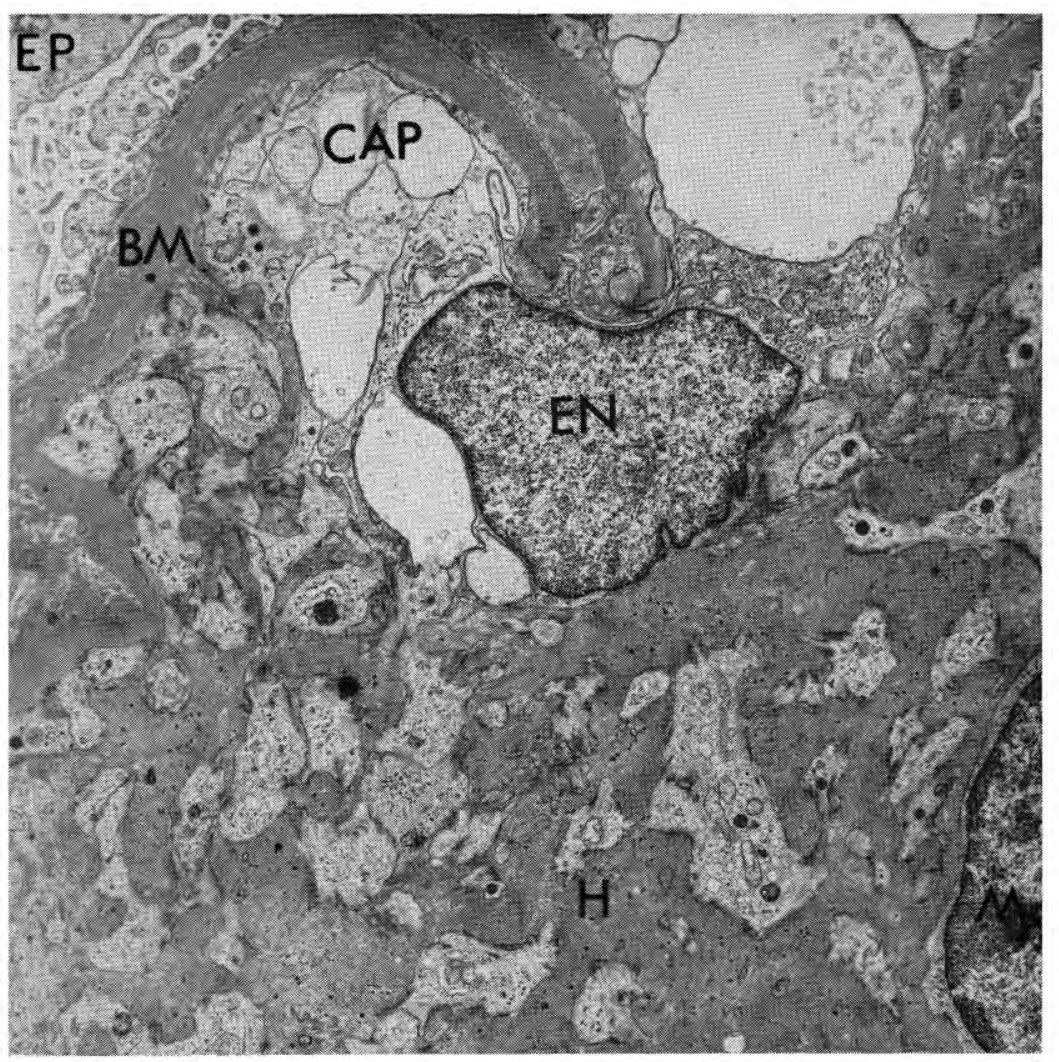

Fig. 2-Electron micrograph of a portion of a glomerulus from a diabetic patient comparable to that shown in Figure 1. The mesangial area has been markedly expanded by large amounts of hyalinoid matrix $(H)$, or basement membrane-like material. The capillary lumina $(C A P)$ are almost completely obliterated by swelling of endothelial cytoplasm and pressure by the hyalinoid matrix. The basement membrane $(B M)$ is markedly thickened. The epithelial cell $(E P)$ foot process structure is distorted and the canaliculi obliterated by fusion of foot processes $(5100 \times)$. 
exudative glomerular lesions, arteriolar sclerosis and thickening of the basement membranes of capillaries and tubules were present. Three long-term dogs also demonstrated diabetic retinopathy and thickening of peripheral capillary basement membranes.

From here on, I would like to speculate about the pathogenesis of these glomerular lesions. I have already stated that I believe the mesangial cells produce the basement membrane material. It is difficult to be sure of this in the normal state, but in the pathological states my colleagues and I are reasonably certain the mesangial cell is producing the hyalinoid matrix which surrounds it, and we have postulated that some of the basement membrane-like material from the mesangial area may migrate out into the periphery of the capillary loop to form its basement mem- brane. Why should the mesangial cell produce excessive quantities of this material? It is our theory that the mesangial cells are damaged or irritated in some way, and that this stimulates the cell not only to proliferate but also to produce more of this basement membrane-like material.

Why should the mesangial cells be irritated? We recently observed in some diabetics black deposits beneath and on both sides of the basement membrane. These appear quite similar to deposits seen in and around the basement membrane in glomerulonephritis and in lupus nephritis. I do not know for sure what they are, but theory says that in glomerulonephritis they probably represent antigen-antibody complexes. There is some evidence to support this. One would assume that in diabetes they might be the same thing. Or they might be some other atypical plasma protein which results from the abnormal pattern of diabetic metabolism. I do not know. I believe this substance may be trapped by the basement membrane. It may be an abnormal substance that is trapped, or it may be an abnormal basement membrane that traps it. As this material is trapped, it is irritating to the mesangial cells and causes them to proliferate and secrete more of the basement membrane-like material. This material gradually fills up the glomeruli, and causes them to become non-functional, ultimately leading to uremia and death. It is of great interest also that these same processes are observed in experimentally-produced diabetes in the dog, a non-hereditary form of diabetes. This would seem to favor a metabolic rather than a genetic origin for the vascular defect.

\section{REFERENCES}

Bloodworth, J. M. B., JR. Diabetic microangiopathy. Diabetes 12: 99 , 1963.

Siperstein, M. D., A. R. Colwell, SR., AND K. MEYER (eds.). Small Blood Vessel Involvement in Diabetes Mellitus. Washington, D. C.: Amer. Inst. Biol. Sci., 1964.

BloodworTh, J. M. B., JR. Basic similarities in pathogenesis of glomerulosclerosis associated with various disease states. Presented to annual meeting of Amer. Diab. Assoc., New York, June 19-20, 1965.

Kimmelstiel, P., and C. Wilson. Intercapillary lesions in the glomeruli of the kidney. Am. J. Path. 12: 83, 1936.

KIMMELSTIEL, P., O. J. KIM, AND J. Beres. Studies on renal biopsy specimens with the aid of the electron microscope. I. Glomeruli in diabetes. Am. J. Clin. Path. 38: 270, 1962.

Bergstrand, A., AND H. Bucht. Electron microscopic investigations on the glomerular lesions in diabetes mellitus (diabetic glomerulosclerosis). Lab. Invest. 6: 293, 1957.
Fig. 3-Light micrograph of a glomerulus' from a diabetic patient showing typical Kimmelstiel-Wilson nodules $(K)$ and diffuse thickening of the remaining mesangial stroma $(430 \times)$. 